\title{
Preventing colorectal cancer in Sri Lanka: step forward
}

\section{Yasara Samarakoon}

Directorate of Non-Communicable Diseases, Ministry of Health and Indigenous Medical Services, Sri Lanka Correspondence: yasara.samarakoon@gmail.com （iD https://orcid.org/0000-0001-6146-9767

DOI: https://doi.org/10.4038/jccpsl.v26i1.8297

Received on 12 March 2020

Accepted on 25 March 2020

\section{Introduction}

Cancers, which origin from the caecum, ascending colon, transverse colon, descending colon, sigmoid colon, recto-sigmoid junction and rectum are included as colorectal cancer and has been recognized as a major cause of mortality and morbidity throughout the world (1).

\section{Global burden of colorectal cancer}

Globally, colorectal cancer is ranked as the third most common cancer in men (age standardized rate (ASR) of 23.6/ 100,000 population) and the second in women (ASR of 12.8/ 100,000 population). As shown in Figure 1 , it is the fourth common cancer among both sexes worldwide (2).

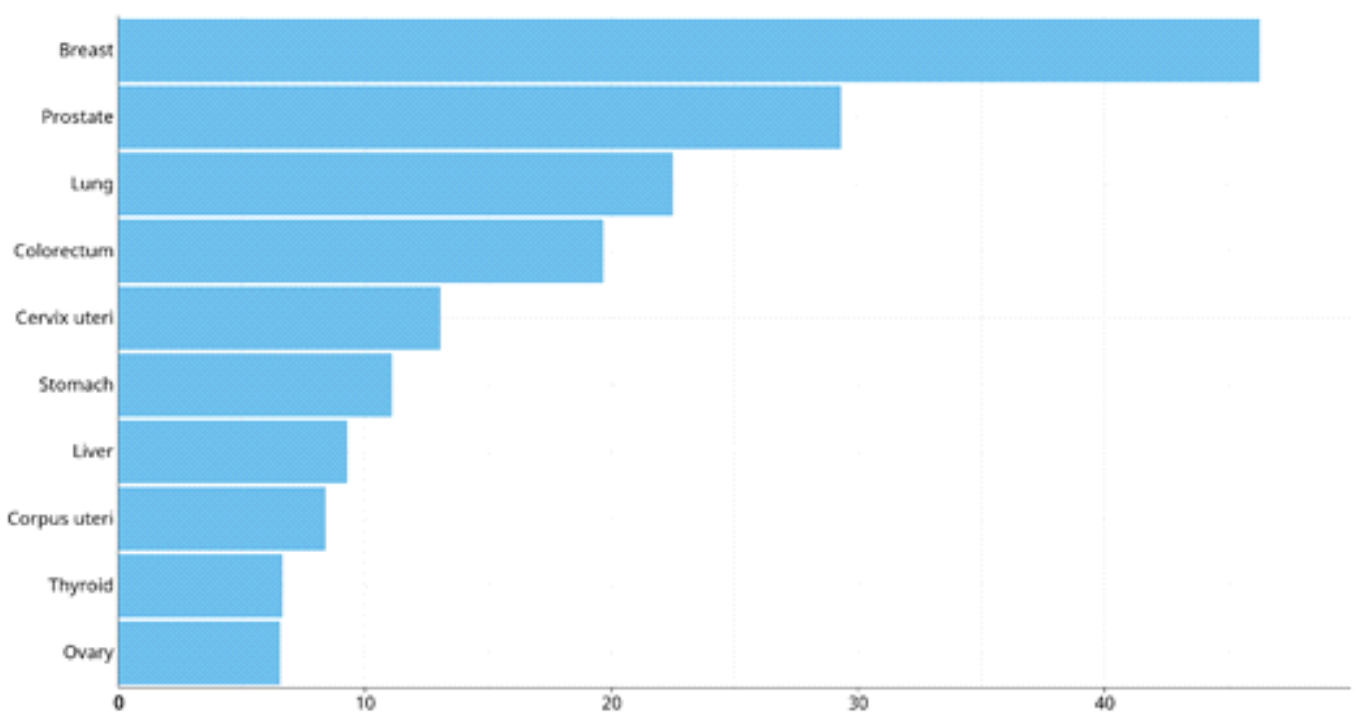

Figure 1. Estimated age standardised incidence rates of cancer in 2018, worldwide among both sexes, all ages

Source: GLOBOCAN 2018, International Agency for Research on Cancer, WHO 
When considering mortality, globally, colorectal cancer was the second leading cause of cancer deaths in 2018 among both sexes (2).

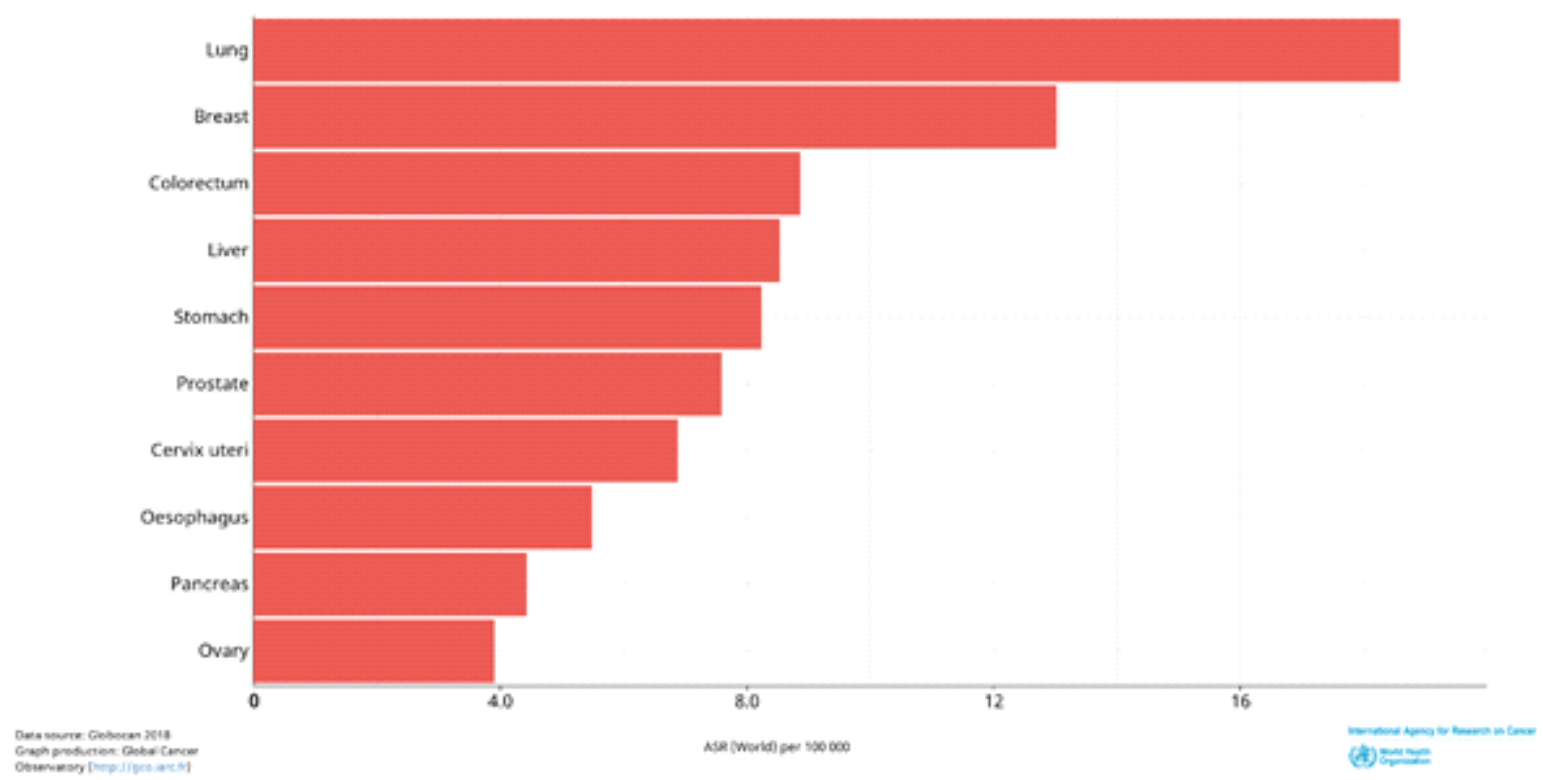

Figure 2. Estimated age standardised mortality rates of cancer in 2018, worldwide among both sexes, all ages

Source: GLOBOCAN 2018, International Agency for Research on Cancer, WHO

\section{Colorectal cancer in Sri Lanka}

Though considered as a disease in the West, the incidence of colorectal cancer in Sri Lanka has increased markedly in recent years. The latest data (2014) indicate that colorectal cancer is ranked as the fourth common cancer among men (ASR of 6.9/ 100,000 population) and the fifth among women (ASR of 6.9/ 100,000 population) (3). The incidence of colorectal cancer has reported an alarming increase, when considering its change over the years (Figure 3).

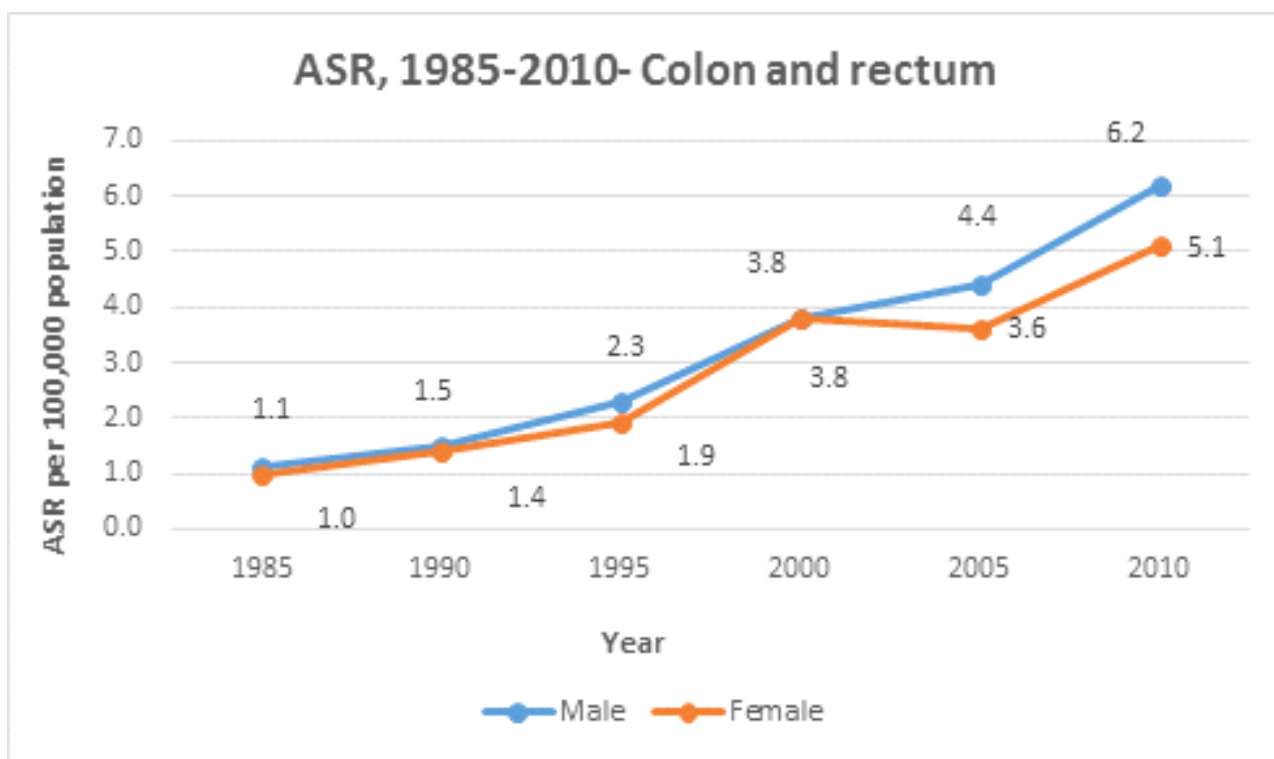

Figure 3. Age standardised incidence rates of colorectal cancer 1985-2010

Source: Cancer incidence data 2010, National Cancer Control Programme, Ministry of Health, Sri Lanka 


\section{Colorectal cancer prevention}

Despite being one of the leading causes of morbidity and mortality worldwide, colorectal cancer is a preventable disease. Several genetic, environmental and lifestyle related risk factors are identified in the pathogenesis of colorectal cancer (Table 1), while many studies have also established a number of protective factors against its development.

Table 1. Risk factors for colorectal cancer

\begin{tabular}{l|l}
\hline Modifiable risk factors & Non-modifiable risk factors \\
\hline Red meat & Increased age \\
Excess alcohol & Male sex \\
Smoking & Family history/known genetic risk \\
Obesity & Inflammatory bowel disease \\
Lack of dietary fibre & \\
Lack of physical activity & \\
\hline
\end{tabular}

Identification of these risk factors and subsequent lifestyle modifications can aid in primary prevention of colorectal cancer. However, several cohort and casecontrol studies investigating the risk factors of colorectal cancer have shown geographical variation. With regards to Sri Lanka, though colorectal cancer is considered a disease of public health importance, there has been no evidence on its risk factors, highlighting the necessity of local evidence which could be applied as primary prevention strategies. On the other, colorectal cancer can largely be prevented by early detection and removal of adenomatous polyps, where survival becomes significantly better when it is diagnosed at an early stage, highlighting the importance of screening.

\section{Colorectal cancer screening}

Secondary prevention via screening has shown to be one of the controversial areas in digestive diseases. The potential for reducing the burden via early detection has become significant due to the slow progression of the disease from detectable precancerous lesions and improved prognosis of patients diagnosed at early stages. There are mainly two goals in colorectal cancer screening programmes. One is to identify and remove precancerous polyps, and thereby reduce its incidence, while the other is to detect disease at an early stage when curative therapy is most likely possible and thereby reduce its mortality.

The argument of colorectal cancer screening is based on the survival rates, which are based on the stage of disease at the time of diagnosis. The five-year survival of an early stage colorectal cancer which has not extended beyond the bowel wall is seen in more than $90 \%$ of the patients. However, it decreases up to $60 \%$ for patients with tumours with lymph node involvement; and to less than $10 \%$ with metastases, highlighting improved survival with early detection and treatment (4).

It is noted that the global incidence and mortality rates of colorectal cancer have significantly declined in the past few decades. This trend is mainly attributed to the adoption of effective screening programmes (5), based on flexible colonoscopy (FS), guaiac-based fecal occult blood test (gFOBT), faecal immunochemical test (FIT) and ccomputed tomographic colonography (CTC).

The FS screens for adenomas as far as in splenic flexure. Randomized trials show favourable results (6-7), with risk reduction of $18 \%$ in incidence and $28 \%$ in deaths related to colorectal cancer (8). Further, FS has a higher detection rate of advanced neoplasia 
compared to gFOBT or FIT, but a lower specificity and sensitivity than colonoscopy for both advanced adenomas and neoplasm (9). Colonoscopy is considered the gold standard for colorectal cancer screening, which could detect and resect neoplasia and precancerous lesions across the entire large bowel. It is relatively safe with recent data suggesting less than 1 per 1000 perforation rate. However, currently there is proof only from observational studies of its superiority over FS in terms of mortality reduction, while cohort studies showing reduction of colorectal cancer incidence up to $90 \%$ has been noted only in symptomatic patients (10). On the other, gFOBT is an inexpensive, simple and widely available test, which demonstrates a relative reduction in colorectal cancer mortality by $15 \%$ (11). Globally, five countries have established gFOBT based screening programs but show lesser uptake (12), most likely due to the nature of the test procedure itself (12). This has resulted in moving to FIT-based screening, which is simpler and easier with fewer fecal samples required. Trials show that FIT has a greater sensitivity for detecting advanced adenomas and colorectal cancer than gFOBT (13). Further, CTC is a rapid radiographic non-invasive imaging test, which requires no sedation and has lower procedural risks compared to colonoscopy (14), but lacks data on its impact on colorectal cancer incidence and mortality. In most countries, colonoscopy is used as the second step in screening after FS or gFOBT (with gFOBT or FIT), as a cost-effective measure for cost and resource constraints (15).

\section{Risk prediction in colorectal cancer}

Despite the wide range of screening options available that could noticeably reduce the risk of colorectal cancer associated mortality, screening rates remain around $60 \%$ since 2010 (16) creating a dilemma in adopting screening programs for colorectal cancer in many countries.

The effectiveness of screening programs could be jeopardized by a multitude of complex factors. These include elevated cost, lack of proper education regarding colorectal cancer, under appreciation of the benefit of screening, a sense of fatalism or simply fear of the screening tests (17). Especially in low-resource settings, it is affected by lack of accessibility, limitations of test performance and sub-optimal screening compliance, which may subsequently result in marked variation in the colorectal cancer incidence and mortality globally (18). Furthermore, the lifetime risk of having colorectal cancer even in a Western country is about $5 \%$ in the population (19). Thus, screening for colorectal cancer would benefit only this $5 \%$, whilst the remaining $95 \%$ would have to undergo this invasive high cost procedure with no personal gain (4). This evidence suggests that it is more efficient to offer colorectal cancer screening using colonoscopy or flexible sigmoidoscopy to high-risk population groups rather than to all as a routine screening test (20). This has prompted many countries to explore the use of high-risk screening for colorectal cancer with appropriate risk stratification of individuals (21). If riskstratified cancer prevention is to be implemented, it requires risk assessment tools that can be used in primary care to identify those most likely to benefit from this intervention (22). Of such tools, risk prediction models which are simple and can be applied in a community setting by a trained person are considered as useful (23).

\section{Prediction of colorectal cancer risk in Sri Lanka}

Samarakoon et al. (2017) conducted a comprehensive scoping review on the available risk models and scores for colorectal cancer, and thereby identify the need for further improvement (24). Out of the 58 risk prediction models identified, most had been developed for advanced colorectal cancer. Most of the articles reviewed were cross-sectional or cohort studies. Statistical methods such as multiple logistic regression was used by a majority, while few have incorporated non-statistical methods such as consensus method and extracting data from published literature. The models have considered 77 different risk factors excluding the genetic variants. Thus, the currently available models have the potential to stratify the general population into risk categories; and allow screening and preventive strategies to be targeted at those most likely to benefit, while leaving those at low risk unexposed to the adverse effects of screening programs.

Clear evidence on its high burden and better survival associated with early detection using colonoscopy or flexible sigmoidoscopy signifies that Sri Lanka will benefit from introduction of a cost effective and affordable screening programme for colorectal cancer. However, being a low- and middle- 
income country, Sri Lanka cannot afford to initiate a national screening program for all above 50 years of age, thus provides flexible sigmoidoscopy or colonoscopy for diagnosing colorectal cancer in patients with symptoms. As an alternative, evidence suggests that a two-step process where population groups at risk of colorectal cancer can be identified using a risk prediction tool is more effective. For this purpose, a risk prediction tool needs to be developed based on the knowledge on country-specific risk factors, in order to stratify and identify those 'being at risk' for whom the colonoscopy or flexible sigmoidoscopy are offered subsequently.

Risk prediction modelling is a mechanism which estimates the probability of an individual having a certain condition based on the presence of multiple risk factors (23). When developing such risk prediction models, obtaining country-specific accurate risk estimates for genetic, environmental and behavioural factors and clinical biological markers identified via cohort or case-control studies is vital (25). Incorporation of variables from published data and expert opinion is another method of selecting the risk predictors (26).

\section{Country-specific risk factors for colorectal cancer}

Samarakoon et al. (2018) further conducted a case-control study among 325 participants (65 incident colorectal cancer cases, 130 hospital and 130 community controls) in five major health care institutions and communities in areas with high incidence in Sri Lanka. Behavioural, genetic and co-morbid risk factors were assessed through an interviewer-administered questionnaire. Risk factors were evaluated using bivariate and multivariate logistic regression.

The results showed that the frequent consumption of red meat (adjusted odds ratio (aOR)=3.06; 95\% $\mathrm{CI}=1.26,7.43)$ and deep fried food $(\mathrm{aOR}=2.54 ; 95 \%$ $\mathrm{CI}=1.22$, 5.39), hypertension for 10 years $(\mathrm{aOR}=3.3$; 95\% CI=1.3, 8.6), colorectal cancer $(\mathrm{aOR}=4.91 ; 95 \%$ $\mathrm{CI}=1.7,14.18)$ and other cancers $(\mathrm{aOR}=3.0 ; 95 \%$ $\mathrm{CI}=1.14,7.81)$ among first degree relatives and age $>50$ years $(\mathrm{aOR}=2.6$; $95 \% \mathrm{CI}=1.1,5.9)$ were significant risk factors compared to hospital controls. Frequent consumption of deep-fried food (aOR=4.2; 95\%
$\mathrm{CI}=1.7,10.1)$, being an ever smoker $(\mathrm{aOR}=3.2 ; 95 \%$ $\mathrm{CI}=1.1,9.3$ ), a current or former drinker $(\mathrm{aOR}=5.4$; $95 \% \mathrm{CI}=1.1,27.8)$ and hypertension for 10 years $(\mathrm{aOR}=5.1 ; 95 \% \mathrm{CI}=1.7,15.6)$ were risk factors compared to community controls (27).

\section{Development and validation of a country specific risk prediction model}

Samarakoon (2016) developed a risk prediction model to estimate the risk of an adult developing colorectal cancer, which was based on the logistic regression model and expert opinion; and further refined with receiver operator characteristic (ROC) curve performance. Assessment of the criterion validity and reliability of the model was performed using a casecontrol design utilizing 65 colorectal cancer new cases and 65 hospital controls aged 30 years or more using an interviewer-administered questionnaire. A risk score was developed for the risk prediction model, by assigning a weighted score for each predictor included in the model. The adjusted ORs were taken as the weighted scores for these predictors, while the other weighted scores were decided based on the pooled OR from meta-analysis from other published literature (28). Table 2 shows the validated risk prediction model with the assigned categories and scores.

The developed and validated model consists of eight predictors with an area under the curve of 0.849 (95\% CI=0.8, 0.9; $\mathrm{p}<0.001$ ). The model demonstrated a sensitivity of $76.9 \%(95 \% \mathrm{CI}=66.7,87.1)$, specificity of $83.1 \%$ (95\% CI=74.0, 92.2), positive predictive value of $82.0 \%(95 \% \mathrm{CI}=72.3,91.6)$ and negative predictive value of $79.3 \%$ (95\% CI=68.5, 88.0) with positive and negative likelihood ratios of 4.6 (95\% $\mathrm{CI}=2.6,7.9)$ and 0.3 (95\% CI=0.2, 0.4); and Kappa coefficient of 0.88 with respect to test re-test reliability.

This model, proven to be valid and reliable among adults aged 30 years and above can be easily administered in a community or a clinical setting by a trained person. It consisted of eight closed-ended questions that can be readily answered by a person when administered through an interviewer. The scoring system to identify those 'at risk' is indicated in the tool for easy use, thus suitable as a screening instrument in Sri Lanka. 
Table 2. Validated risk prediction model with the assigned categories and scores

\begin{tabular}{llc}
\hline Predictor variable & Categories & Score \\
\hline Age & Less than 50 years & 0 \\
& 50 years or more & 3 \\
\hline
\end{tabular}

Frequent consumption of deep-fried food (3 times or more) per week for the period of last 20 years and beyond
Rare or never: less than 3 times

per week or never 0

Frequent: 3 or more times per week

Frequent consumption of red meat (3 times or more) Rare or never: less than 3 times per week for the period of 20 years and beyond per week or never $\quad 0$

Frequent: 3 or more times per week

Diagnosis of colorectal cancer at or before 60 years No among first degree relatives Yes

Diagnosis of other cancer at or before 60 years No

(breast, endometrial, ovary) among first degree relatives

Yes

Personal history of intestinal polyps diagnosed No

before 10 years (histologically confirmed)

Yes

3

Personal history of hypertension for more than 10 years (medically confirmed)

No

0

Yes

3

Diagnosis of inflammatory bowel disease before 10 years

No

0

(histologically confirmed)

Yes

\section{Prevalence of the population 'at risk' of developing colorectal cancer in Sri Lanka}

Samarakoon et al. (2017) also estimated the prevalence of population at risk of developing colorectal cancer based on the validated risk prediction model developed for Sri Lanka.

A community-based cross-sectional, descriptive study was conducted among a representative sample of 811 adults aged 30 years and above in the districts of Colombo and Gampaha, selected using a multi-stage cluster sampling technique. The validated risk prediction model was used in the form of an interviewer-administered questionnaire. The prevalence of those 'at risk' of colorectal cancer was assessed based on the validated cut-off score, while they were further divided as 'moderate' and 'high' risk based on cut-off values agreed upon by the experts.

The age-adjusted prevalence of those 'at risk' was $12.5 \%$ (95\% CI=12.3, 12.7). Age-adjusted prevalence of those at 'moderate' and 'high' risk were 11.8\% (95\% $\mathrm{CI}=11.6,12.0)$ and $0.72 \%(95 \% \mathrm{CI}=0.7,0.8)$, respectively. The high prevalence indicates the public health importance of the problem and the necessity for screening for colorectal cancer in Sri Lanka. The prevalence also highlights the logistical difficulties in offering the follow-up diagnostic colonoscopy examinations to those screened positive. Considering the logistic difficulties in offering follow-up diagnostic colonoscopy examinations for all, the study recommends that those found to be at 'high-risk' to be referred for colonoscopy (29). 


\section{Future perspectives and recommendations}

Addressing dietary risk factors for colorectal cancers, mainly long-term frequent consumption of deep-fried food and red meat is recommended as a primary preventive measure. Noting that these foods are discouraged in the existing national dietary guidelines and school canteen policy, the evidence recommends strengthening their implementation. Furthermore, other co-morbid conditions such as hypertension, should be addressed by measures to prevent such conditions. Adopting healthy lifestyles, specifically healthy dietary practices of less salt and more fiber can be considered as specific recommendations, which can be strengthened in primary care setting.

Sri Lanka offers free healthcare services and has initiated healthy lifestyle centres (HLCs) at the lowest level of primary care institutions since 2011 to offer adults structured non-communicable disease screening (30). More than 900 such centres are distributed throughout the country at present. The main service objective of HLCs is to reduce the risk of NCDs of 40-65-year-old adults by early detection of risk factors and to improve the access for specialized care of those found to be at high-risk.

With the availability of a local risk prediction model, the scope of HLC can be extended for early detection of colorectal cancer in Sri Lanka. This prevalence of being 'at risk' denotes the burden of colorectal cancer on the health system in future. Such an initiation should be accompanied by informing the general public about the importance of getting their risk estimated, so that they could undergo FS or colonoscopy examination if required to diagnose the condition early, enabling successful treatment. With regards to persons identified as being 'at risk', they should be directed for diagnostic colonoscopy examination and further management, while those at 'moderate risk' should be advised on simple screening tests such as FOBTs or modifying the risk factors at primary care level. A further step in introducing a screening program in Sri Lanka is establishing a referral system for those 'at risk'. This information would form the basis to advocate for a policy decision to evaluate the need for a screening program among the high-risk population during HLCs in Sri Lanka.

\section{References}

1. Curado MP, Edwards B, Shin HR, Storm H, Ferlay J, Heanue M, Boyle P. Cancer incidence in five continents. Vol. IX. Lyon, France: International Agency of Research in Cancer, 2007.

2. GLOBOCAN 2018. Cancer Today 2018. Geneva: International Agency for Research on Cancer, World Health Organization. Available from: http://gco.iarc.fr/ today/online-analysis.

3. Ministry of Health. Cancer incidence data Sri Lanka 2014. Colombo: Ministry of Health, 2018.

4. Bretthauer, M. Colorectal cancer screening. Journal of Internal Medicine 2011; 270, 87-98.

5. Siegel R, Desantis C, Jemal A. Colorectal cancer statistics. CA: a Cancer Journal for Clinicians 2014; 64(2): 104-117.

6. Holme Ø, Løberg M, Kalager M, et al. Effect of flexible sigmoidoscopy screening on colorectal cancer incidence and mortality: a randomized clinical trial. JAMA2014; 312: 606-615.

7. Atkin WS, Edwards R, Kralj-Hans I, et al. Once-only flexible sigmoidoscopy screening in prevention of colorectal cancer: a multicentre randomised controlled trial. Lancet 2010; 375: 1624-1633.

8. Brenner H, Stock C, Hoffmeister M. Effect of screening sigmoidoscopy and screening colonoscopy on colorectal cancer incidence and mortality: systematic review and meta-analysis of randomised controlled trials and observational studies. BMJ 2014; 348: g246.

9. Bevan R \& Rutter MD. Colorectal cancer screeningWho, How and When? Clinical Endoscopy 2018; 51(1): 37-49.

10. Winawer SJ, Zauber AG, Ho MN, et al. Prevention of colorectal cancer by colonoscopic polypectomy. New England Journal of Medicine 1993; 329: 1977-1981.

11. Hewitson P, Glasziou P, Irwig L, Towler B, Watson E. Screening for colorectal cancer using the faecal occult blood test, Hemoccult. The Cochrane Database of Systematic Reviews 2007; (1): CD001216.

12. Palmer CK, Thomas MC, von Wagner C, Raine R. Reasons for non-uptake and subsequent participation in the NHS bowel cancer screening programme: a qualitative study. British Journal of Cancer 2014; 110: 1705-1711.

13. Allison JE, Fraser CG, Halloran SP, Young GP. Population screening for colorectal cancer means getting FIT: the past, present, and future of colorectal cancer screening using the faecal immunochemical test for haemoglobin (FIT). Gut and Liver 2014; 8: 117-130. 
14. Ranasinghe I, Parzynski CS, Searfoss R, Montague J, Lin Z, Allen J, Vender R, Bhat K, Ross JS, Bernheim S, et al. Differences in colonoscopy quality among facilities: development of a post-colonoscopy riskstandardized rate of unplanned hospital visits. Gastroenterology 2016; 150: 103-113.

15. Schreuders EH, Grobbee EJ, Spaander MC, Kuipers EJ. Advances in faecal tests for colorectal cancer screening. Current Treatment Options in Gastroenterology 2016; 14: 152-162.

16. Sabatino SA, White MC, Thompson TD, Klabunde $\mathrm{CN}$; Centers for Disease Control and Prevention (CDC). Cancer screening test use - United States, 2013. MMWR Morbidity and Mortality Weekly Report 2015; 64: 464-468.

17. Brenner AT, Ko LK, Janz N, Gupta S, Inadomi J. Race/ ethnicity and primary language: health beliefs about colorectal cancer screening in a diverse, low-income population. Journal of Health Care for the Poor and Underserved 2015; 26: 824-838.

18. Torre LA, Bray F, Siegel RL, Ferlay J, Lortet-Tieulent J, Jemal A. Global cancer statistics, 2012. CA: a Cancer Journal for Clinicians 2015; 65: 87-108.

19. GLOBOCAN 2012. Colorectal cancer: estimated incidence, mortality and prevalence worldwide in 2012, World Health Organization. Available from: http://globocan.iarc.fr/Pages/fact_sheets_ cancer.aspx\#

20. Boyle P \& Leon ME. Epidemiology of colorectal cancer. British Medical Bulletin 2002; 64: 1-25.

21. Nelson RS \& Thorson AG. Colorectal cancer screening. Current Oncology Reports 2009; 11(6): 482489.

22. Armstrong R, Hall BJ, Doyle J, Waters E. Scoping the scope of a Cochrane review. Journal of Public Health (Oxford, England) 2011; 33: 147-150.
23. Win AK, Macinnis RJ, Hopper JL, Jenkins MA. Risk prediction models for colorectal cancer: a review. Cancer Epidemiology, Biomarkers and Prevention 2011; 21(3): 398-410.

24. Samarakoon YM, Pathmeswaran A, Gunawardena NS. Risk prediction models for colorectal cancer: a scoping review. Journal of Postgraduate Institute of Medicine 2017; 4(2): E561-24.

25. Freedman AN, Seminara D, Gail MH, Hartge P, Colditz GA, Ballard-Barbash R, et al. Cancer risk prediction models: a workshop on development, evaluation and application. Journal of the National Cancer Institute 2005; 97(10): 715-723.

26. Kumari PBVR. Risk factors and risk assessment of breast cancer among women in the district of Colombo. MD Thesis (Community Medicine). Colombo: University of Colombo, 2013.

27. Samarakoon YM, Gunawardena NS, Pathirana A. Behavioural, familial and comorbid illness risk factors of colorectal cancer: a case control study. Ceylon Medical Journal 2018; 63(3): 139.

28. Samarakoon YM. Risk factors and risk prediction of colorectal cancer among adults in the districts of Colombo and Gampaha. MD Thesis (Community Medicine). Colombo: University of Colombo, 2016.

29. Samarakoon YM, Gunawardena NS, Pathirana A. Prevalence of the population 'at risk' of developing colorectal cancer in Sri Lanka. Journal of Consultant Community Physicians Sri Lanka 2017; 23 (4).

30. Mallawaarchchi DSV, Wickramasinghe SC, Somatunga LC, Siriwardana VTSK, Gunawardena NS. Healthy lifestyle centres: a service for screening noncommunicable diseases through primary health care institutions in Sri Lanka. WHO South-East Asia Journal of Public Health 2016; 5(2): 89-95. 